\title{
Differential expression of claudin-4, occludin, SOX2 and proliferating cell nuclear antigen between basaloid squamous cell carcinoma and squamous cell carcinoma
}

\author{
FUYUKI SATO ${ }^{1}$, UJJAL K. BHAWAL ${ }^{2}$, ITARU TOJYO ${ }^{3}$, SHIGEYUKI FUJITA ${ }^{3}$, \\ SHIN-ICHI MURATA ${ }^{4}$ and YASUTERU MURAGAKI ${ }^{1}$
}

\author{
${ }^{1}$ Department of Pathology, Wakayama Medical University School of Medicine, Wakayama 641-8509; \\ ${ }^{2}$ Department of Biochemistry and Molecular Biology, Nihon University School of Dentistry at Matsudo, \\ Chiba 271-8587; Departments of ${ }^{3}$ Oral and Maxillofacial Surgery, and ${ }^{4}$ Human Pathology, \\ Wakayama Medical University, Wakayama 641-8509, Japan
}

Received January 25, 2019; Accepted May 24, 2019

DOI: $10.3892 / \mathrm{mmr} .2019 .10417$

\begin{abstract}
Basaloid squamous cell carcinomas (BSCCs) in oral lesions are extremely rare, and the histology is not well understood. Histologically, they are often similar to conventional squamous cell carcinoma (SCC). The present study was designed with an aim to distinguish BSCC from SCC using claudin-4, occludin, SRY-box 2 (SOX2) and proliferating cell nuclear antigen (PCNA) immunoreactivities and staining patterns. Three BSCCs (with abundant, with moderate, and without squamous components) specimens and 20 SCC specimens were selected for comparison of their immunoreactivity. These specimens were stained with claudin-4, occludin, SOX2 and PCNA. In addition to histological analysis, the expression of claudin-4, occludin and PCNA was determined in oral cancer HSC 2 and HSC 3 cells with or without SOX 2 overexpression, and cell proliferation was determined by XTT assay. Claudin-4 had strong and occludin had weak immunoreactivity as detected in the membrane of squamous components of BSCC but not in cancer cells. No obvious detection of squamous components and cancer cells were observed in SCC. SOX2 and PCNA immunoreactivities in SCC had dot-like staining patterns in the nuclei of partial and marginal cancer cells. In contrast, in BSCCs, SOX2 and PCNA had diffuse staining patterns in almost all cancer cells. SOX2 overexpression had little effect on the expression levels of claudin-4, occludin and PCNA. It also had little effect on the cell proliferation of HSC2 and HSC3 cells. Differences in immunoreactivity and staining pattern may be valuable to distinguish between BSCC and SCC in diagnosis.
\end{abstract}

Correspondence to: Dr Fuyuki Sato, Department of Pathology, Wakayama Medical University School of Medicine, 811-1 Kimiidera, Wakayama 641-8509, Japan

E-mail: fsatodec1dec2@yahoo.co.jp

Key words: basaloid squamous cell carcinoma, claudin-4, occludin, SOX2, PCNA

\section{Introduction}

Basaloid squamous cell carcinomas (BSCCs) in oral lesions are extremely rare and are a variant of squamous cell carcinoma (SCC) (1-4). Although BSCCs are reportedly aggressive, have early lymph node metastasis, and have worse prognosis, their biological features are similar to those of conventional SCC (1-4). Several studies have demonstrated that BSCCs have grossly flat or slightly elevated mucosa and histologically small crowded cells with hyperchromatic nuclei and stromal hyalinization (1-4). BSCC exhibits comedonecrosis and has a palisading pattern of basal cells with squamous components in the center, although not specifically $(3,5,6)$. As the histological aspects of BSCC are often similar to those of SCC, it is difficult to distinguish BSCC from SCC for diagnosis, especially in biopsy specimens. It has also been reported that BSCCs exhibit high proliferation involving proliferating cell nuclear antigen (PCNA) and Ki-67 immunoreactivity (7-9). Interestingly, cell adhesion molecule E-cadherin and $\beta$-catenin expression patterns reportedly differ between BSCC and SCC (9). There are few reports that feature tight junction molecule analysis of claudin- 4 and occludin in SCC $(10,11)$. Their immunoreactivities were reported to be extremely weak in cancer cells $(10,11)$. Whether claudin- 4 and occludin are expressed in BSCC, however, remains unknown. We hypothesized that cell proliferation, adhesion and tight junction markers facilitate a differential diagnosis. In the present study, the immunohistochemical detection of cell proliferation marker PCNA, tight junction markers claudin-4 and occludin, and stem cell marker SOX2 was conducted in BSCC and SCC. Additionally, it was ascertained whether SOX 2 overexpression affects the expression of these factors and cell proliferation.

\section{Materials and methods}

Tissue preparation. Histological specimens collected from January 2014 to December 2018 were retrieved from our hospital's archives according to the guidelines of the 
Japanese Society of Pathology. Informed consent was provided by each patient for the use of the clinical images. Two cases of BSCC and 20 cases of conventional SCC were collected from craniocervical lesions, and each case included lymph node metastasis. Specimens were obtained from the Department of Diagnostic Pathology, Wakayama Medical University. Another BSCC specimen was obtained from the Department of Pathology, Tsurumi University School of Dental Medicine. Diagnosis was performed by at least two pathologists. Clinical and pathological information is described below. Case 1 is a female patient, 81 years of age, with a clinically diagnosed mandibular malignant tumor; pathological diagnosis is BSCC with abundant squamous components. Case 2 is a male patient, 57 years of age, with a clinically diagnosed malignant tumor in the palatine tonsil; pathological diagnosis is BSCC with moderate squamous components and lymph node (LN) metastasis. Case 3 is a male patient, 68 years of age, with clinically diagnosed tongue cancer; pathological diagnosis is BSCC without squamous components. Case 4 is a female patient, 87 years of age, with clinically diagnosed gingival cancer; pathological diagnosis is well-differentiated SCC. Case 5 is a male patient, 78 years of age, with clinically diagnosed tongue cancer; pathological diagnosis is well-differentiated SCC and LN metastasis. Case 6 is a female patient, 74 years of age, with clinically diagnosed buccal cancer; pathological diagnosis is poorly differentiated SCC. The clinical information regarding the other SCC cases is documented in Table I. Partial resection and LN dissection specimens were used in cases 2 and 5, and biopsy specimens were used in the other cases.

Immunohistochemistry. Claudin-4, occludin, SOX2 and PCNA expression in BSCC and SCC tissues were evaluated from serial deparaffinized sections. Our specimens had been fixed with formalin from 24 to $48 \mathrm{~h}$ at room temperature and treated with routine processing as in a previous study (12). Ten 4-mm serial sections were prepared for staining and were incubated with primary antibodies for $2 \mathrm{~h}$. Immunohistochemistry was performed using a Discovery Auto-Stainer with automated protocols (Ventana Medical Systems, Inc.; Roche) as previously described (13). The average number of PCNA-positive cells from case 1 to case 23 were counted in 10 random microscopic fields at 400 magnification. The SOX2 intensity was determined by qualitative assessment of three levels as weak, 1; moderate, 2; and strong, 3 . We defined a diffuse staining pattern as that positively detected in almost all cancer cells but a dot-like staining pattern as that positively detected in partial and marginal cancer cells.

Cell culture and treatment. HSC2 and HSC 3 human oral squamous cell carcinoma cells, which do not have basaloid characteristics were obtained from the Health Science Research Resources Bank (Osaka, Japan). These cells were cultured in Dulbecco's modified Eagle's medium (DMEM) (Sigma Chemical Co.; Merck KGaA) supplemented with $10 \%$ fetal bovine serum and $1 \%$ penicillin streptomycin antibiotics. The construct expression vector for SOX2 was used for transfection. The human SOX2 plasmid (26817) was purchased from Addgene. Transient plasmid transfection was performed as previously described (14).

Western blot analysis. HSC2 cells were lysed using M-PER lysis buffer (Thermo Fisher Scientific, Inc.). Protein determination was performed using the bicinchoninic method. A total of $40 \mu \mathrm{g}$ protein was loaded in each lane. The total cell lysates were run on $14 \%$ SDS-polyacrylamide gels followed by western blotting using standard procedures. The proteins were transferred on to PVDF membrane. For blocking, membranes were incubated with $5 \%$ skim milk for $60 \mathrm{~min}$ at room temperature after protein transfer. A WesternBright Sirius kit (Advansta) was used for antibody detection, and an AE-9300 Ez capture MG (ATTO) was used for image data capture. We repeated the western blot analysis three times and the results were similar.

Antibodies. The following commercial antibodies were purchased: claudin-4 (1:100, mouse monoclonal, sc-376643; Santa Cruz Biotechnology Inc.), occludin (1:100, mouse monoclonal, sc-133255; Santa Cruz Biotechnology), SOX2 (1:100, rabbit polyclonal, Ab97959, Abcam), PCNA (1:1,000, mouse monoclonal, sc-56; Santa Cruz Biotechnology) and actin (1:10,000, mouse monoclonal, A5441; Sigma Chemical Co.; Merck KGaA).

Real-time (quantitative) PCR ( $q P C R$ ). We prepared three independent RNA samples from HSC3 cells. Total RNA was isolated and first-strand cDNA was synthesized as previously described (13). Real-time PCR was performed using SYBR Green Master Mix (Bio-Rad Laboratories, Inc.). The thermocycling conditions were the following: Initial denaturation at $95^{\circ} \mathrm{C}$ for $3 \mathrm{~min}$, followed by 40 cycles of $95^{\circ} \mathrm{C}$ for $10 \mathrm{sec}$ and $60^{\circ} \mathrm{C}$ for $30 \mathrm{sec}$. The $2^{-\Delta \Delta C q}$ method was used for relative quantification (15). Amplification primer sequences were designed as follows: SOX2-F, 5'-GAATGCCTTCAT GGTGTGGT-3' and R, 5'-TTGCTGATCTCCGAGTTGTG-3'; claudin-4-F, 5'-ATGGCCTCCATGGGGCTACA-3' and R, 5'-AGCGAGTCGTACACCTTGCA-3'; occludin-F, 5'-GCG GATTGGTTTATCTTGGA-3' and R, 5'-CTGGATGACATG GCTGATTG-3'; PCNA-F, 5'-AGGTGTTGGAGGCACTC AAG-3' and R, 5'-AGTCCATGCTCTGCAGGTTT-3'; and $18 \mathrm{~S}$ rRNA-F, 5'-GCGCCGCTAGAGGTGAAAT-3' and R, 5'-GAA AACATTCTTGGCAAATGCTT-3'. Data were normalized using $18 S$ rRNA. qPCR was repeated three times and the results were similar.

Cell proliferation assay. HSC2 and HSC 3 cells were seeded into 96-well plates. After transfection, $50 \mathrm{ml}$ of XTT kit reaction solution was added to each well (XTT-based) (Biological Industries) and the cells were incubated at $37^{\circ} \mathrm{C}$ for an additional $2 \mathrm{~h}$ according to the manufacturer's instructions. Absorbance at optical density at $480 \mathrm{~nm}\left(\mathrm{OD}_{480}\right)$ and at $\left(\mathrm{OD}_{650}\right)$ was measured using a 96 -well microplate reader (SH-9000; Hitachi).

Statistical analysis. Statistical analysis with Dunnett's test was performed using JMP Pro software version 13.0 (SAS Institute Japan, Tokyo, Japan). The data are expressed as the mean value \pm SE (bars) of three independent samples. ${ }^{*} \mathrm{P}<0.05$ 
Table I. Immunohistochemical detection of SOX2 and PCNA in BSCC and SCC specimens.

\begin{tabular}{|c|c|c|c|c|c|c|}
\hline Case & Age & Sex & Lesion & Diagnosis & SOX2 intensity & PCNA-positive cells \\
\hline 1 & 81 & $\mathrm{~F}$ & Mandibular & $\mathrm{BSCC}$ & 3 & 113 \\
\hline 2 & 57 & M & Tonsil & BSCC & 3 & 358.6 \\
\hline 3 & 68 & $\mathrm{M}$ & Tongue & BSCC & 3 & 224.2 \\
\hline 4 & 87 & $\mathrm{~F}$ & Gingiva & $\mathrm{SCC}(\mathrm{w})$ & 2 & 48.2 \\
\hline 5 & 78 & M & Tongue & $\operatorname{SCC}(w)$ & 3 & 51.2 \\
\hline 6 & 74 & $\mathrm{~F}$ & Buccal & $\operatorname{SCC}(p)$ & 1 & 33.4 \\
\hline 7 & 87 & M & Gingiva & $\mathrm{SCC}(\mathrm{w})$ & 2 & 62.4 \\
\hline 8 & 85 & M & Gingiva & $\mathrm{SCC}(\mathrm{p})$ & 2 & 37.3 \\
\hline 9 & 57 & $\mathrm{M}$ & Tongue & $\mathrm{SCC}(\mathrm{w})$ & 1 & 27.6 \\
\hline 10 & 86 & M & Gingiva & $\mathrm{SCC}(\mathrm{m})$ & 2 & 41.4 \\
\hline 11 & 85 & M & Gingiva & $\mathrm{SCC}(\mathrm{m})$ & 2 & 41.2 \\
\hline 12 & 69 & $\mathrm{M}$ & Maxillary & $\mathrm{SCC}(\mathrm{p})$ & 2 & 50.9 \\
\hline 13 & 64 & $\mathrm{M}$ & Tongue & $\mathrm{SCC}(\mathrm{m})$ & 3 & 47.6 \\
\hline 14 & 73 & $\mathrm{~F}$ & Tongue & $\mathrm{SCC}(\mathrm{m})$ & 2 & 73 \\
\hline 15 & 87 & $\mathrm{~F}$ & Oral floor & $\mathrm{SCC}(\mathrm{m})$ & 2 & 52.4 \\
\hline 16 & 89 & $\mathrm{~F}$ & Gingiva & $\mathrm{SCC}(\mathrm{w})$ & 3 & 56.7 \\
\hline 17 & 69 & $\mathrm{M}$ & Tongue & $\mathrm{SCC}(\mathrm{w})$ & 2 & 47 \\
\hline 18 & 90 & $\mathrm{~F}$ & Gingiva & $\mathrm{SCC}(\mathrm{w})$ & 3 & 41.9 \\
\hline 19 & 53 & $\mathrm{M}$ & Tongue & $\mathrm{SCC}(\mathrm{w})$ & 3 & 94.8 \\
\hline 20 & 66 & $\mathrm{~F}$ & Tongue & $\mathrm{SCC}(\mathrm{m})$ & 2 & 81.2 \\
\hline 21 & 60 & $\mathrm{M}$ & Oral floor & $\mathrm{SCC}(\mathrm{m})$ & 3 & 68.4 \\
\hline 22 & 69 & M & Oral floor & $\mathrm{SCC}(\mathrm{m})$ & 3 & 77.2 \\
\hline 23 & 65 & M & Oral floor & $\mathrm{SCC}(\mathrm{p})$ & 3 & 77 \\
\hline
\end{tabular}

SOX2 intensity was classified as follows: 1 , weak; 2, moderate; 3, strong. PCNA-positive cells were calculated as the average number of PCNA-positive cells in 10 random microscopic fields at 400x magnification. BSCC, basaloid squamous cell carcinoma; SCC, squamous cell carcinoma; PCNA, proliferating cell nuclear antigen; SOX2, SRY-box 2; w, well differentiated; m, moderately differentiated; p, poorly differentiated.

was considered to indicate a statistically significant result, and ANOVA was used followed by Dunnett's test.

\section{Results}

Macroscopic and radioscopic aspects in BSCC. Representative macroscopic and radioscopic aspects in case 1 of BSCC are shown in Fig. 1. Fig. 1A shows flat appearance of gingival mucosa in a right anterior lesion. Fig. 1B shows the huge mandibular swelling that was found. Diffuse swelling was found in the palatine tonsil or tongue in case 2 and 3, respectively (data not shown). An orthopantomograph of the BSCC at first medical examination showed a multilocular cyst surrounded by well-defined radiolucent borders (Fig. 1C, left panel). The patient was treated with radiotherapy and the orthopantomograph at 8 months after the first examination showed a decreased multilocular cyst with calcification (Fig. 1C, right panel). This indicated that radiotherapy was effective. The other two patients with BSCC were not treated with radiotherapy.

Histological aspects of BSCC and SCC. Fig. 2A shows representative histological hematoxylin and eosin (H\&E)-stained images from case 1, 2 and 3 of BSCC. Fig. 2B shows representative histological images from case 4,5 and 6 of SCC.

Immunohistochemical detection of claudin-4, occludin, SOX 2 and PCNA in BSCC and SCC. Representative images of claudin-4, occludin, SOX2 and PCNA immunoreactivities for case 1, 2 and 3 of BSCC are shown in Fig. 3. Claudin-4 immunoreactivity in case 1 was strongly detected in the cell membrane of squamous components, whereas no obvious detection was observed in cancer cells. Claudin-4 immunoreactivity in case 2 of the primary tumor was weakly detected in the membrane of squamous components, whereas no obvious detection was observed in the cancer cells. No obvious detection of claudin- 4 immunoreactivity in case 2 was observed in cancer cells of LN metastasis. Claudin-4 immunoreactivity in case 3 was not detected in the cancer cells. Occludin immunoreactivity was similar to claudin- 4 as above, but in case 1 it was weakly detected when compared to claudin-4. We defined diffuse staining patterns as positively detected in almost all cancer cells but dot-like staining patterns as positively detected in partial and marginal cancer cells. SOX2 in case 1 had a diffuse staining pattern in the nucleus, cytoplasm and in the membrane of squamous components. SOX 2 immunoreactivity 
A

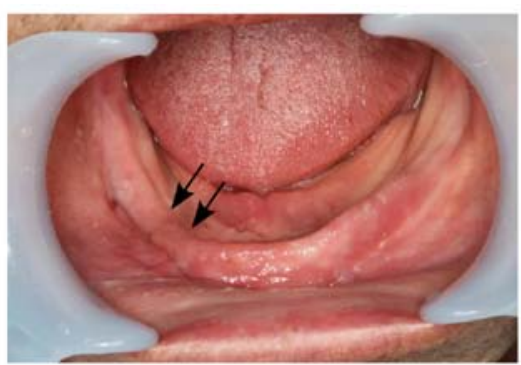

C

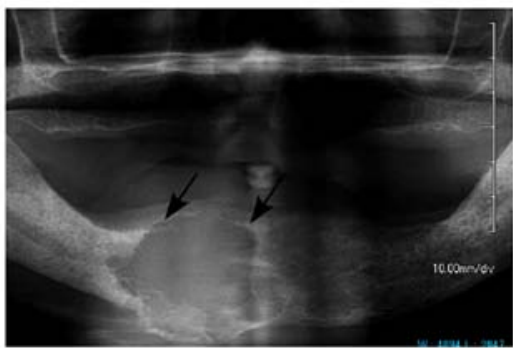

B
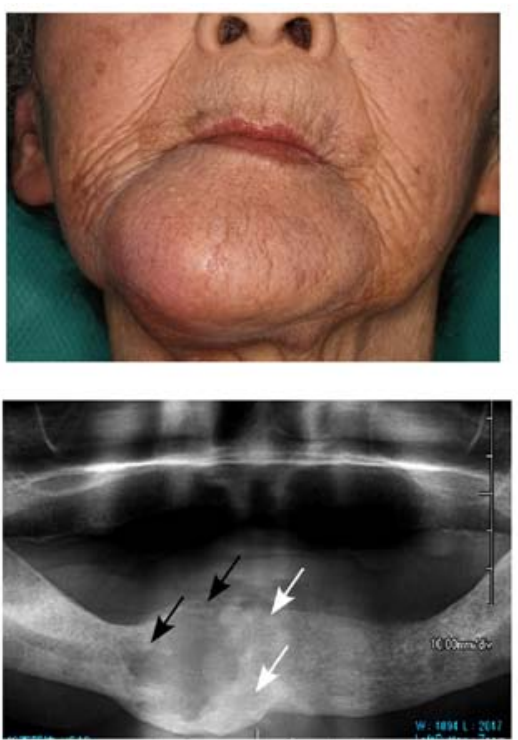

Figure 1. Clinical features of BSCC. (A) Appearance of flat gingival mucosa in the right mandibular (arrows). (B) Mandibular swelling. (C) The radiographical presentation is that of an extensive and well-defined multiocular radiolucency (left panel, black arrows) in right anterior lesion of mandibular. An orthopantomograph at the second medical examination (8 months after the initial examination) showing decreased multiocular cyst with calcification (right panel, white arrows). BSCC, basaloid squamous cell carcinoma.

A

BSCC

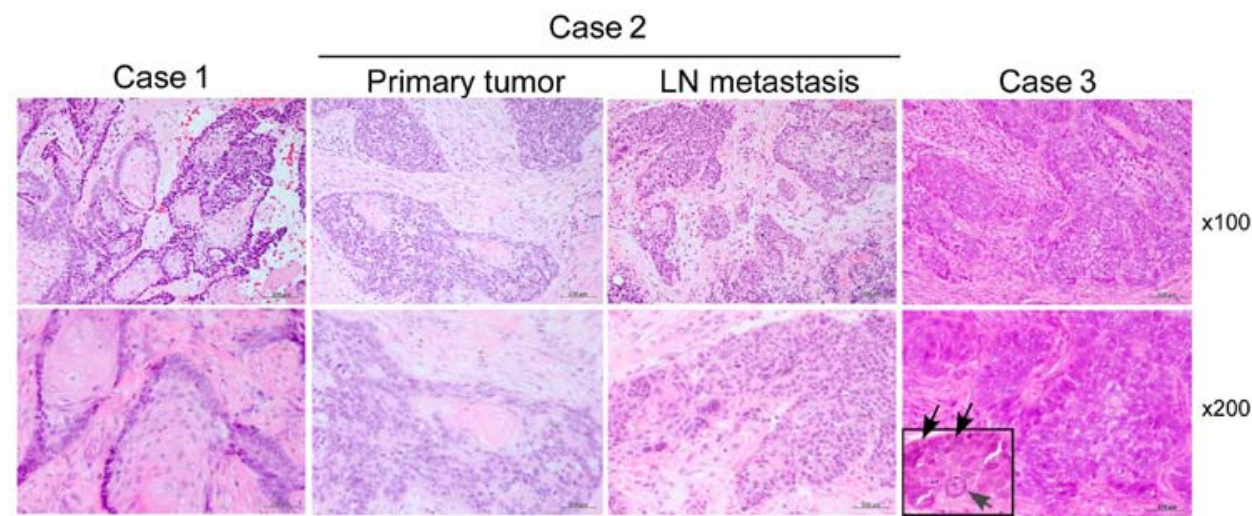

B

SCC

Case 5

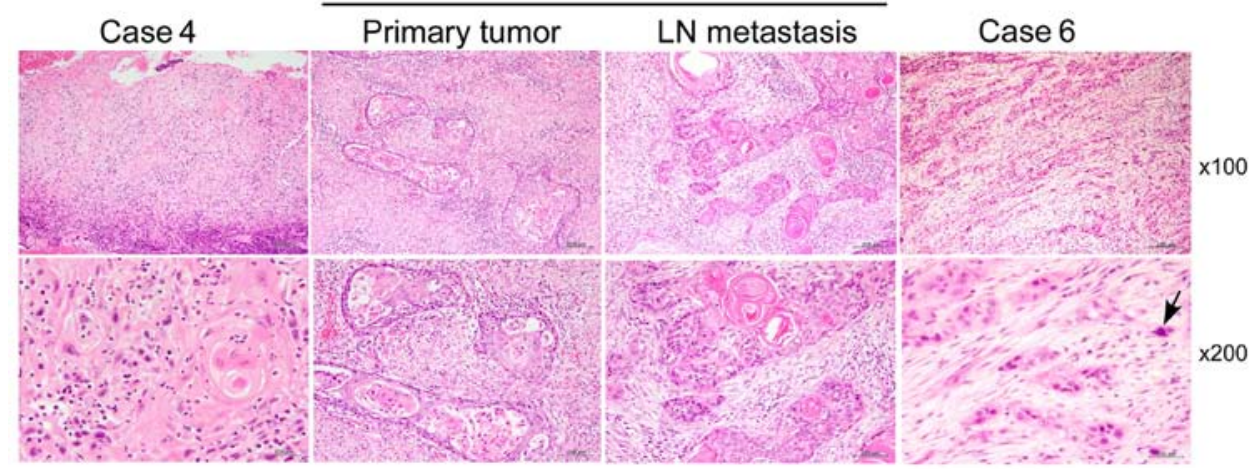

Figure 2. Histological features of BSCC and SCC. (A) Representative H\&E images of case 1, 2 and 3 of BSCC. Case 1, BSCC with abundant squamous components. Case 2, BSCC with moderate squamous components. Case 2 includes primary tumor and lymph node (LN) metastasis. Case 3, BSCC without squamous components. Top panels shows x100 magnification and bottom panels show x200 magnification (scale bars, $200 \mathrm{~mm}$ ). Square inset shows a larger image. Black arrows show a palisading pattern in the basal cells, and the gray arrow shows large atypical nucleus. (B) Representative H\&E images of case 4, 5, and 6 of SCC. Case 4 , SCC with abundant squamous components. Case 5, SCC with abundant squamous components of primary tumor and LN metastasis. Case 6, SCC without squamous components. There are no palisading pattern in case 6, but a large atypical nucleus is observed (black arrow). Top panel shows x100 magnification and bottom panel shows x200 magnification (scale bars, 200 mm). BSCC, basaloid squamous cell carcinoma; SCC, squamous cell carcinoma; H\&E, hematoxylin and eosin. 


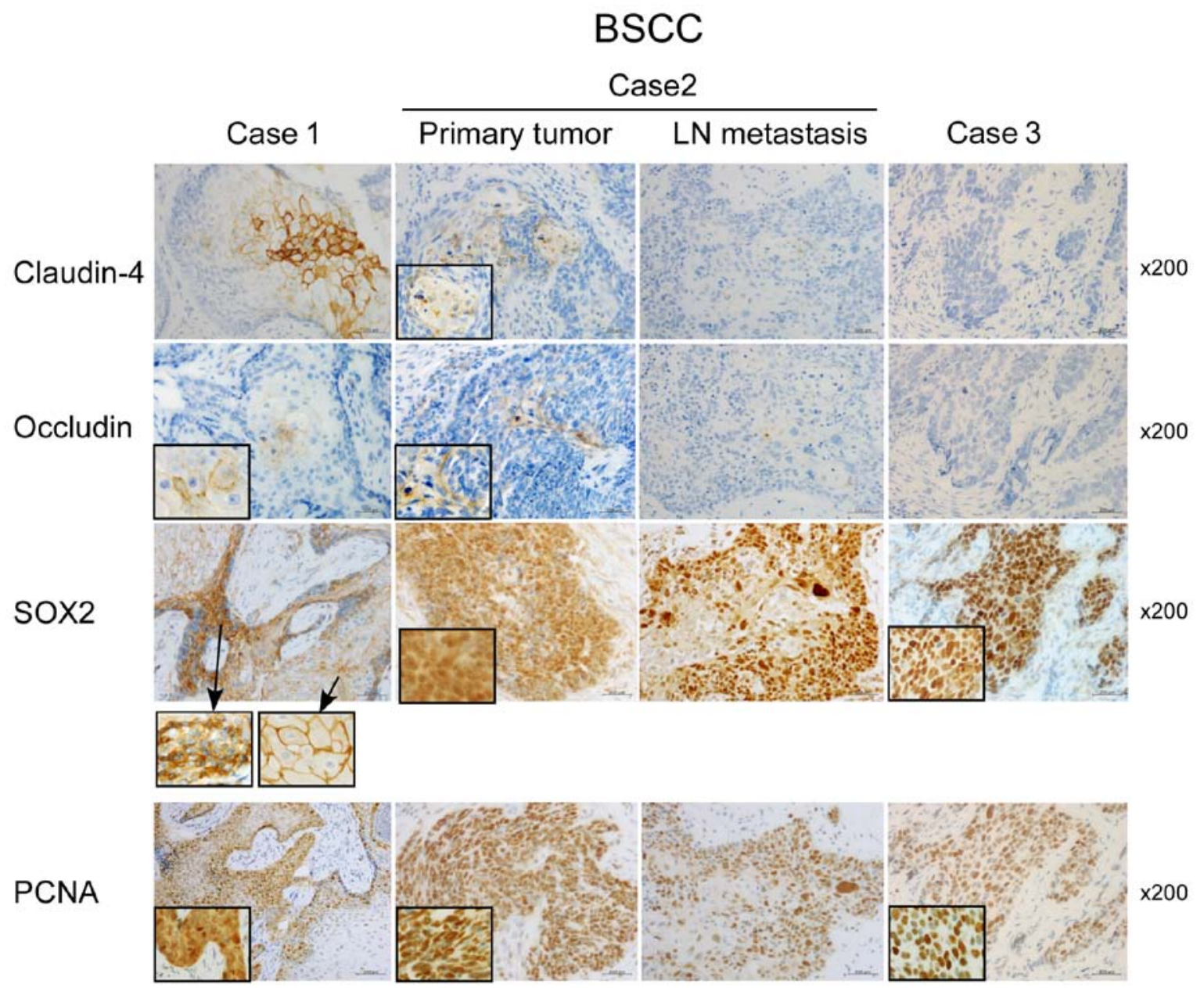

Figure 3. Immunoreactivities of claudin-4, occludin, SOX2 and PCNA in BSCC. Representative images for the immunoreactivities of claudin-4, occludin, SOX2 and PCNA in case 1, 2 and 3 of BSCC. Each panel shows x200 magnification (scale bars, $200 \mathrm{~mm}$ ). Each square inset shows larger immunostaining images. The square in SOX2 shows larger immunostaining images in the nucleus and cytoplasm, and in the membrane, respectively. The square in PCNA shows larger immunostaining images in the nucleus. BSCC, basaloid squamous cell carcinoma; SOX2, SRY-box 2; PCNA, proliferating cell nuclear antigen.

in cases 2 and 3 of the primary tumor and LN metastasis exhibited a diffuse staining pattern in the nucleus and some positive staining in squamous components was observed. The PCNA immunoreactivity of the primary tumor and LN metastasis in case 1,2 and 3 also had diffuse staining in the nucleus, but there was no obvious detection observed in squamous components.

Representative images of claudin- 4 , occludin, SOX 2 and PCNA immunoreactivities in SCC are shown in Fig. 4A. No positive immunoreactivities for claudin- 4 and occludin in the primary tumor and $\mathrm{LN}$ metastasis from cases 4, 5 and 6 were detected in either cancer cells or squamous components. SOX2 immunoreactivities from case 4, 5 and 6 were dot-like staining patterns in the nucleus of cancer cells. No obvious detection was observed in squamous components. PCNA immunoreactivity from case 4, 5 and 6 of the primary tumor was similar to that of SOX2. There appeared to be weaker detection of PCNA immunoreactivity in case 6 compared with case 4 and 5. SOX2 intensities and PCNA-positive cells of BSCC and SCC cases are provided in Table I and Fig. 4B. The numbers of PCNA-positive cells were higher in BSCC compared to SCC. SOX2 intensities in BSCC were all strong, but they exhibited a variation in SCC.

SOX2 overexpression exhibits little effect on the expression of claudin-4, occludin and PCNA. SOX2 expression was strongly detected in all cases of BSCC. Therefore, it was ascertained whether SOX2 overexpression affects expression of claudin-4, occludin and PCNA using oral SCC cancer HSC2 cells. SOX2 overexpression had little effect on the protein expression of occludin and PCNA (Fig. 5A). Endogenous protein expression of claudin- 4 was not detected in HSC 2 cells, thus expression levels were further examined by qPCR in HSC3 cells. SOX2 overexpression had little effect on the mRNA expression of claudin-4, occludin and PCNA in HSC3 cells (Fig. 5B).

SOX2 overexpression exhibits little effect on cell proliferation. Abnormalities in SOX2 expression affect proliferation, migration and apoptosis in various types of cancer cells. It was thus ascertained whether SOX2 overexpression affects cell proliferation of oral cancer HSC 2 and HSC 3 cells. SOX 2 overexpression had little effect on the cell proliferation of HSC2 and HSC 3 cells at 24 and 48 h (Fig. 5C). 

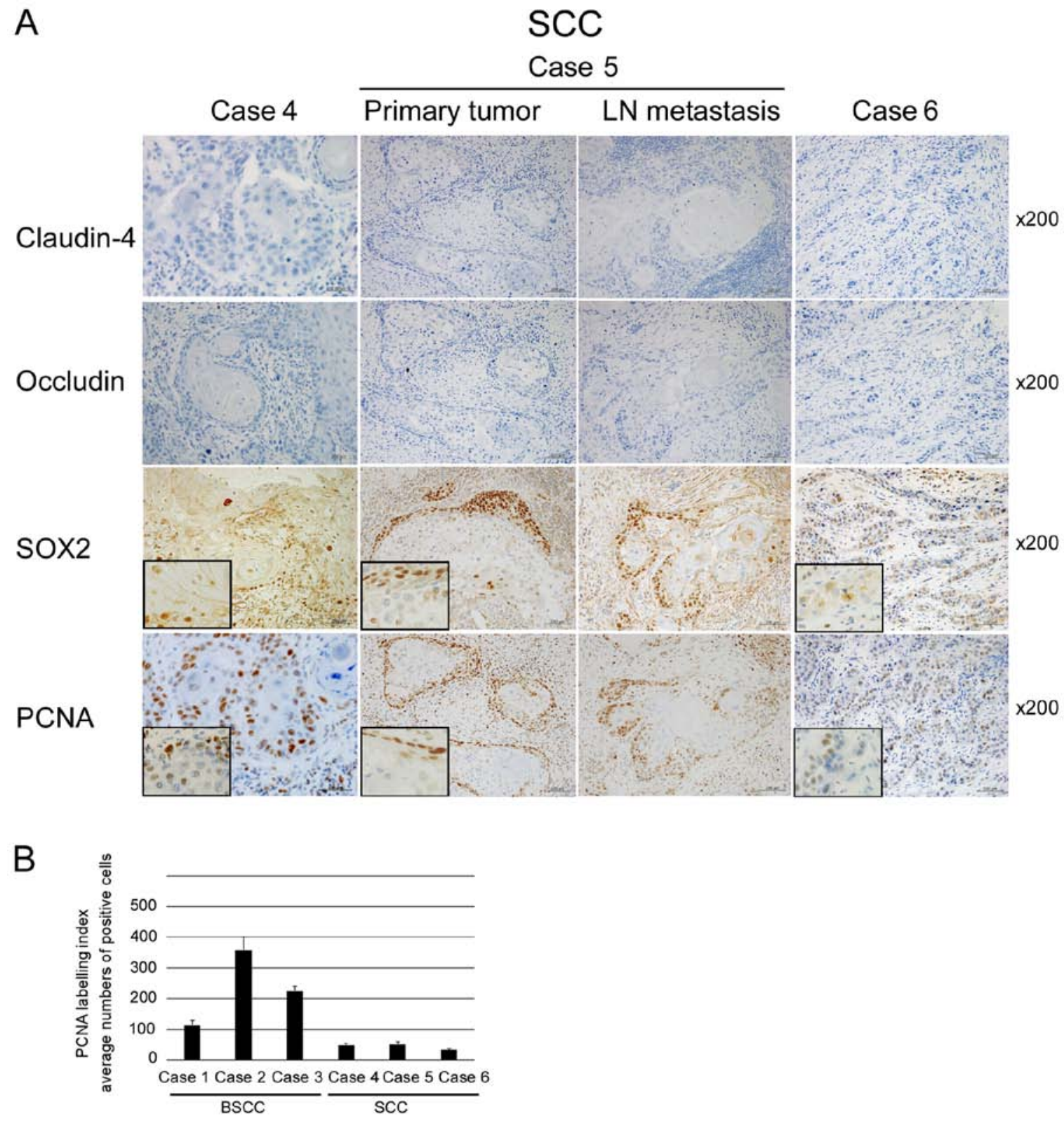

Figure 4. Immunoreactivities of claudin-4, occludin, SOX2 and PCNA in SCC. (A) Representative images of the immunoreactivities of claudin-4, occludin, SOX2 and PCNA in case 4, 5 and 6 of SCC. Each panel shows x 200 magnification (scale bars, $200 \mathrm{~mm}$ ). Each square shows larger immunostaining images of SOX2 and PCNA. (B) The average number of PCNA-positive cells in cases 1-6 in 10 random microscopic fields at $x 400$ magnification. SCC, squamous cell carcinoma; SOX2, SRY-box 2; PCNA, proliferating cell nuclear antigen.

\section{Discussion}

Basaloid squamous cell carcinoma (BSCC) is regarded as a high-grade malignant tumor, yet it remains unclear how to distinguish it from squamous cell carcinoma (SCC). Oral BSCC is extremely rare, thus it is important to understand both the clinical and histological aspects. At our university, a diagnosed of oral BSCC has been made only in 2 cases during the past 20 years. Moreover, in another university, only 1 case of BSCC was found during the past 20 years. Therefore, more than 3 cases of BSCC could not be obtained, making additional cases difficult to find. Articles reporting on oral BSCC are quite rare, and there are certain studies that have used only 2 BSCC cases (16-18). We will attempt use more BSCC cases, collaborating with other universities in future research.
Clinically, BSCC is highly sensitive to radiotherapy $(4,19)$. SCC, on the other hand, is not sensitive. In case 1, radiotherapy induced the suppression of tumor growth and calcification in the mandible. This clinical aspect is compatible with BSCC.

The histological aspects of BSCC are similar to SCC. Takubo et al (20) reported that a hyaline substance positively stained with periodic acid-Shiff (PAS) staining had been observed in BSCC of the esophagus. In the present study, however, we did not obviously observe positive staining of PAS in BSCC (data not shown). In addition, there are no critical markers for distinguishing between the two. Therefore, immunohistochemical detection of claudin-4, occludin, SOX2 and PCNA was conducted in BSCC compared to SCC. Overexpression of claudins has been reported in several types of cancer, and the overexpression or downregulation of 


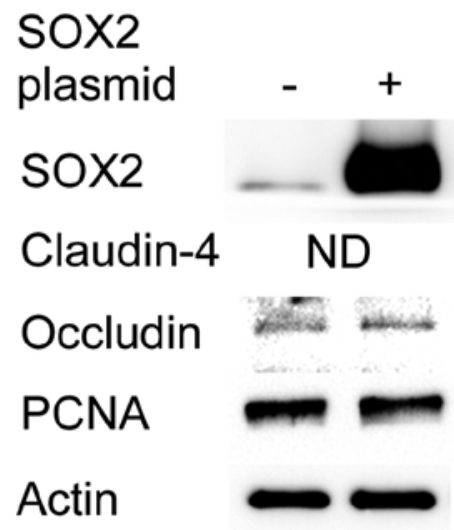

B
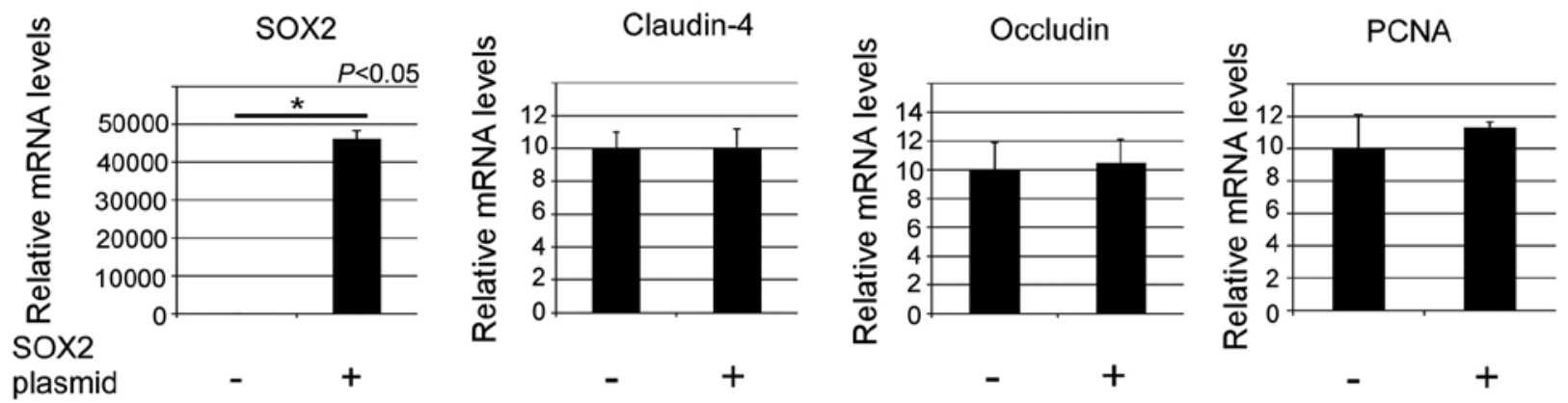

C

HSC2
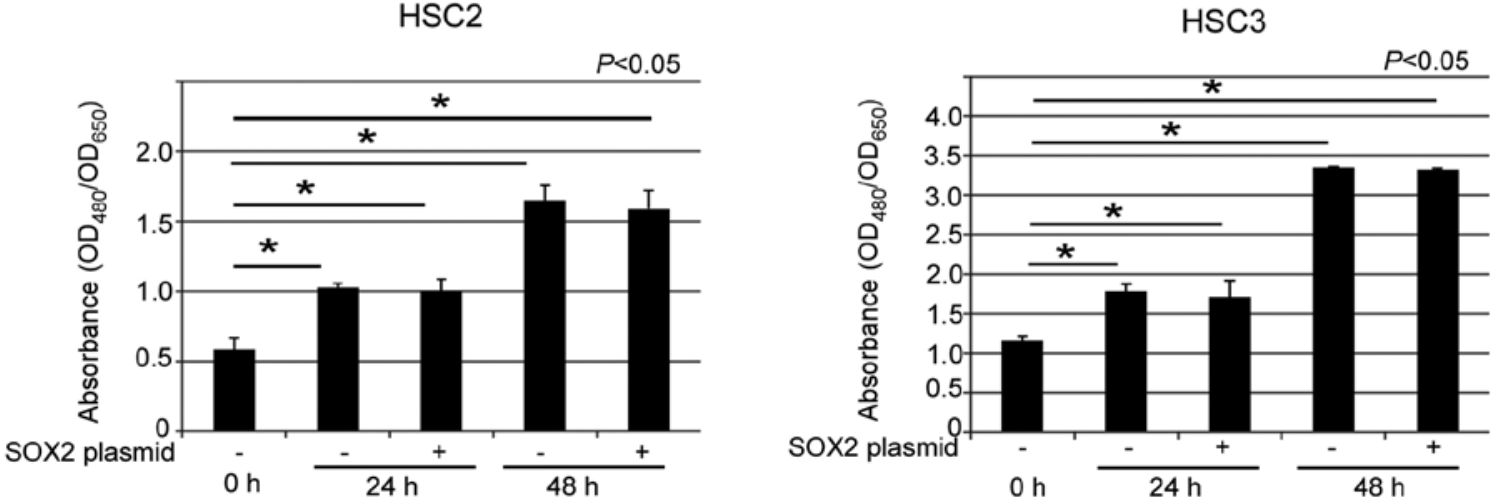

Figure 5. SOX2 overexpression in oral cancer HSC2 and HSC 3 cells. (A) Representative western blotting images of SOX2, occludin, PCNA and actin in HSC2 cells with (+) or without (-) SOX2 overexpression. ND for claudin-4 means not detected. The western blot analysis was repeated three times and similar results were obtained. (B) Relative SOX2, claudin-4, occludin and PCNA mRNA expression in HSC3 cells with (+) or without (-) SOX2 overexpression. Data are expressed as mean values $\pm \mathrm{SE}$ (bars) of three independent samples. ${ }^{*} \mathrm{P}<0.05$, as determined using t-test. qPCR was repeated three times and similar results were obtained. (C) Cell proliferation of $\mathrm{HSC} 2$ and $\mathrm{HSC} 3$ cells with (+) or without (-) SOX2 overexpression was determined. The absorbance $\left(\mathrm{OD}_{480} / \mathrm{OD}_{650}\right)$ at 0,24 , and $48 \mathrm{~h}$ is shown. Data are expressed as mean values $\pm \mathrm{SE}$ (bars) of three independent samples. ${ }^{*} \mathrm{P}<0.05$, as determined using Dunnett's test. SOX2, SRY-box 2; PCNA, proliferating cell nuclear antigen.

claudins and occludin are associated with tumor progression and recurrence (21-24). Therefore, whether claudin- 4 and occludin are candidate markers for BSCC was ascertained. It is worth noting that the immunoreactivities of claudin-4 and occludin in BSCC were detected in the membrane of squamous components. They were not observed in cancer cells of BSCC without squamous components in case 3. This implies that claudin- 4 and occludin play important roles in squamous components of BSCC. The squamous components are created by keratinization. Claudin- 4 and occludin may therefore regulate the adhesion of keratinization in BSCC. On the contrary, no obvious detection of claudin- 4 and occludin immunoreactivities in SCC were observed in either squamous components or cancer cells. It has been reported that claudin-4 immunoreactivity in SCC is weakly or not significantly detected in the membrane of cancer cells. Considering this, it was speculated that the functions of claudin- 4 and occludin in squamous components of BSCC differ from those of SCC. The 
differences in immunoreactivities between BSCC and SCC are valuable for differential diagnosis. However, these differences are not useful in BSCC without squamous components. Future research must clarify this possibility by using claudins with more cases of BSCC.

The staining patterns of PCNA immunoreactivity between BSCC and SCC were also found to be different. The PCNA labeling index is over $50 \%$ in BSCC and is associated with the malignant feature of BSCC (25). In SCC, the PCNA immunoreactivities exhibited dot-like staining patterns in nuclei, whereas in BSCC they appeared as diffuse staining patterns in the nuclei. Sampaio-Góes et al (7) and Yu et al (25) also reported findings that corroborate ours. Our specimens were fixed with formalin from 24 to $48 \mathrm{~h}$. It has been reported that formalin fixation for over 24 and $48 \mathrm{~h}$ ensures better results for immunoreactivity (26). Thus, our samples used for immunoreactivity analysis were maintained in optimum conditions.

SOX2 overexpression is a poor prognostic factor and is associated with tumor progression (27-31). SOX2 regulates matrix metalloproteinase (MMP)-2, MMP-3, vimentin, slug, E-cadherin and $\beta$-catenin (31-33). Whether SOX2 plays a significant role in oral cancer, however remains unclear. We found that SOX2 immunoreactivity in SCC was presented in a dot-like staining pattern, but in BSCC this was exhibited as a diffuse staining pattern. SOX2 immunoreactivity in BSCC in case 1 was also observed in the membrane of squamous components. We therefore examined whether SOX2 overexpression affects the expression of claudin- 4 and occludin, but it had little effect. This implies that claudin-4 and occludin overexpression may occur independently of SOX2. Finally, the effect of SOX2 overexpression on cell proliferation was determined, involving PCNA expression. No significant effect was observed implying that other factors, such as c-Myc, may be associated with cell proliferation of oral cancer.

Human papillomavirus (HPV) affects the progression, recurrence and radiotherapy sensitivity of tonsillar and posterior tongue SCC $(34,35)$. In addition, the protein expression of angiogenesis-related proteins and TNF-receptors differ between HPV-positive and -negative tissues (34). Although our BSCC sample from case 2 was from the tonsil, the p16 immunoreactivity was negative. It is possible that the observed immunoreactivities of case 2 may not be due to HPV infection. Our tongue SCC cases were from a side margin lesion; however, future studies needs to clarify whether the HPV status affects progression and immunoreactivities.

Poorly differentiated SCC has no squamous components, thus it is extremely difficult to distinguish poorly differentiated SCC from BSCC without squamous components. In this study, it was demonstrated that the staining patterns of SOX2 and PCNA immunoreactivities in BSCC and SCC were different. SOX2 and PCNA presented as dot-like staining, appearing as partially marginal staining in cancer cells of SCC, whereas they were diffuse staining in almost all cancer cells of BSCC. On the other hand, there were no differences in claudin- 4 and occludin immunoreactivities. These diffuse staining patterns of SOX2 and PCNA suggest that BSCC has more aggressive and proliferative potential than SCC. It has been reported that the diffuse staining pattern of NOTCH1 is correlated with mutation status and poor prognosis in adenoid cystic carcinoma (36). Therefore, diffuse staining patterns of SOX2 and PCNA may correlate with BSCC mutation and progression.

This observation may be useful for additional diagnosis. In conclusion, claudin-4 and occludin immunoreactivities and staining patterns of SOX2 and PCNA may be utilized to potentially carry out a differential diagnosis between BSCC and SCC

\section{Acknowledgements}

The authors would like to thank Dr Yumi Ito from the Department of Pathology, Tsurumi University School of Dental Medicine, who supplied a BSCC specimen.

\section{Funding}

The present study was supported by JSPS KAKENHI grant no. 16K09624 (FS), and by Nihon University Multidisciplinary Research Grant for 2018 (UKB).

\section{Availability of data and materials}

The datasests used and/or analyzed during the current study are available from the corresponding author on reasonable request.

\section{Authors' contributions}

FS and UKB performed experiments, carried out the pathological diagnosis and completed the draft. FS, SIM and YM performed the pathological diagnosis. IT and SF performed the clinical diagnosis and submitted clinical images and materials. UKB, IT, SF, SIM and YM revised manuscript. All authors read and approved the final manuscript.

\section{Ethics approval and consent to participate}

This study was approved by the Wakayama Medical University Research Ethics Committee (Protocol no. 1715) and histological specimens were retrieved from our hospital archives. Informed consent was provided by each patient for the use of the clinical images.

\section{Patient consent for publication}

The patient provided written informed consent for the publication of clinical images.

\section{Competing interests}

The authors declare that they have no competing interests.

\section{References}

1. Fritsch VA, Gerry DR and Lentsch EJ: Basaloid squamous cell carcinoma of the oral cavity: An analysis of 92 cases. Laryngoscope 124: 1573-1578, 2014.

2. Thariat J, Badoual C, Faure C, Butori C, Marcy PY and Righini CA: Basaloid squamous cell carcinoma of the head and neck: Role of HPV and implication in treatment and prognosis. J Clin Pathol 63: 857-866, 2010. 
3. Ereño C, Gaafar A, Garmendia M, Etxezarraga C, Bilbao FJ and López JI: Basaloid squamous cell carcinoma of the head and neck: A clinicopathological and follow-up study of 40 cases and review of the literature. Head Neck Pathol 2: 83-91, 2008.

4. Shen W, Sakamoto N and Yang L: Cause-specific mortality prediction model for patients with basaloid squamous cell carcinomas of the head and neck: A competing risk analysis. J Cancer 9: 4009-4017, 2018.

5. Wain SL, Kier R, Vollmer RT and Bossen EH: Basaloid-squamous carcinoma of the tongue, hypopharynx, and larynx: Report of 10 cases. Hum Pathol 17: 1158-1166, 1986.

6. Luna MA, el Naggar A, Parichatikanond P, Weber RS and Batsakis JG: Basaloid squamous carcinoma of the upper aerodigestive tract. Clinicopathologic and DNA flow cytometric analysis. Cancer 66: 537-542, 1990.

7. Sampaio-Góes FC, Oliveira DT, Dorta RG, Nonogaki S, Landman G, Nishimoto IN and Kowalski LP: Expression of PCNA, p53, Bax, and Bcl-X in oral poorly differentiated and basaloid squamous cell carcinoma: Relationships with prognosis. Head Neck 27: 982-989, 2005.

8. Coletta RD, Cotrim P, Vargas PA, Villalba H, Pires FR, de Moraes M and de Almeida OP: Basaloid squamous carcinoma of the oral cavity: Report of 2 cases and study of AgNOR, PCNA, p53, and MMP expression. Oral Surg Oral Med Oral Pathol Oral Radiol Endod 91: 563-569, 2001

9. Pereira $\mathrm{CH}$, Morais MO, Martins AF, Soares MQ, Alencar Rde C, Batista AC, Leles CR and Mendonça EF: Expression of adhesion proteins (E-cadherin and $\beta$-catenin) and cell proliferation (Ki-67) at the invasive tumor front in conventional oral squamous cell and basaloid squamous cell carcinomas. Arch Oral Biol 61: 8-15, 2016.

10. Sappayatosok K and Phattarataratip E: Overexpression of claudin-1 is associated with advanced clinical stage and invasive pathologic characteristics of oral squamous cell carcinoma. Head Neck Pathol 9: 173-180, 2015.

11. Phattarataratip E and Sappayatosok K: Expression of claudin-5, claudin-7 and occludin in oral squamous cell carcinoma and their clinico-pathological significance. J Clin Exp Dent 8: e299-e306, 2016.

12. Wu Y, Sato F, Yamada T, Bhawal UK, Kawamoto T, Fujimoto K, Noshiro M, Seino H, Morohashi S, Hakamada K, et al: The BHLH transcription factor DEC1 plays an important role in the epithelial-mesenchymal transition of pancreatic cancer. Int J Oncol 41: 1337-1346, 2012.

13. Sato F, Kohsaka A, Takahashi K, Otao S, Kitada Y, Iwasaki Y and Muragaki Y: Smad3 and Bmal1 regulate $\mathrm{p} 21$ and S100A4 expression in myocardial stromal fibroblasts via TNF- $\alpha$. Histochem Cell Biol 148: 617-624, 2017.

14. Sato F, Muragaki Y and Zhang Y: DEC1 negatively regulates AMPK activity via LKB1. Biochem Biophys Res Commun 467: 711-716, 2015

15. Livak KJ and Schmittgen TD: Analysis of relative gene expression data using real-time quantitative PCR and the 2(-Delta Delta C(T)) method. Methods 25: 402-408, 2001.

16. Peddapell K, Rao GV, Sravya T and Ravipati S: Basaloid squamous cell carcinoma: Report of two rare cases and review of literature. J Oral Maxillofac Pathol 22: 285, 2018.

17. Heera R, Ayswarya T, Padmakumar SK and Ismayil P: Basaloid squamous cell carcinoma of oral cavity: Report of two cases. J Oral Maxillofac Pathol 20: 545, 2016.

18. Patel PN, Mutalik VS, Rehani S and Radhakrishnan R: Basaloid squamous cell carcinoma of oral cavity with incongruent clinical course. BMJ Case Rep 2013: bcr 2013200441, 2013.

19. Maebayashi T, Ishibashi N, Aizawa T, Sakaguchi M, Taku H, Ohhara M, Takimoto T and Tanaka Y: A long-surviving patient with advanced esophageal basaloid squamous cell carcinoma treated only with radiotherapy: Case report and literature review. BMC Gastroenterol 17: 151, 2017.
20. Takubo K, Mafune K, Tanaka Y, Miyama T and Fujita K Basaloid-squamous carcinoma of the esophagus with marked deposition of basement membrane substance. Acta Pathol Jpn 41: 59-64, 1991.

21. Resnick MB, Konkin T, Routhier J, Sabo E and Pricolo VE: Claudin-1 is a strong prognostic indicator in stage II colonic cancer: A tissue microarray study. Mod Pathol 18: 511-518, 2005.

22. Sheehan GM, Kallakury BV, Sheehan CE, Fisher HA and Kaufman RP Jr and Ross JS: Loss of claudins-1 and -7 and expression of claudins- 3 and -4 correlate with prognostic variables in prostatic adenocarcinomas. Hum Pathol 38: 564-569, 2007.

23. Lourenço SV, Coutinho-Camillo CM, Buim ME, Pereira CM, Carvalho AL, Kowalski LP and Soares FA: Oral squamous cell carcinoma: Status of tight junction claudins in the different histopathological patterns and relationship with clinical parameters. A tissue-microarray-based study of 136 cases. J Clin Pathol 63 : 609-614, 2010.

24. Martin TA, Mansel RE and Jiang WG: Loss of occludin leads to the progression of human breast cancer. Int J Mol Med 26: 723-734, 2010

25. Yu GY, Gao Y, Peng X, Chen Y, Zhao FY and Wu MJ: A clinicopathologic study on basaloid squamous cell carcinoma in the oral and maxillofacial region. Int J Oral Maxillofac Surg 37: 1003-1008, 2008

26. Patil S, Rao RS, Ganavi BS and Majumdar B: Natural sweeteners as fixatives in histopathology: A longitudinal study. J Nat Sci Biol Med 6: 67-70, 2015.

27. Wang H, Zhou Y, Liu Q, Xu J and Ma Y: Prognostic value of SOX2, Cyclin D1, p53, and ki-67 in patients with esophageal squamous cell carcinoma. Onco Targets Ther 11: 5171-5181, 2018

28. Wuebben EL, Wilder PJ, Cox JL, Grunkemeyer JA, Caffrey T, Hollingsworth MA and Rizzino A: SOX2 functions as a molecular rheostat to control the growth, tumorigenicity and drug responses of pancreatic ductal adenocarcinoma cells Oncotarget 7: 34890-34906, 2016.

29. Chung JH, Jung HR, Jung AR, Lee YC, Kong M, Lee JS and Eun YG: SOX2 activation predicts prognosis in patients with head and neck squamous cell carcinoma. Sci Rep 8: 1677, 2018.

30. Yang N, Hui L, Wang Y, Yang H and Jiang X: SOX2 promotes the migration and invasion of laryngeal cancer cells by induction of MMP-2 via the PI3K/Akt/mTOR pathway. Oncol Rep 31: 2651-2659, 2014.

31. Girouard SD, Laga AC, Mihm MC, Scolyer RA, Thompson JF, Zhan Q, Widlund HR, Lee CW and Murphy GF: SOX2 contributes to melanoma cell invasion. Lab Invest 92: 362-370, 2012

32. Liu X, Qiao B, Zhao T, Hu F, Lam AK and Tao Q: Sox 2 promotes tumor aggressiveness and epithelial-mesenchymal transition in tongue squamous cell carcinoma. Int J Mol Med 42: 1418-1426, 2018.

33. Han X, Fang X, Lou X, Hua D, Ding W, Foltz G, Hood L, Yuan Y and Lin B: Silencing SOX2 induced mesenchymal-epithelial transition and its expression predicts liver and lymph node metastasis of CRC patients. PLoS One 7: e41335, 2012.

34. Ramqvist T, Näsman A, Franzén B, Bersani C, Alexeyenko A, Becker S, Haeggblom L, Kolev A, Dalianis T and Munck-Wikland E: Protein expression in tonsillar and base of tongue cancer and in relation to human papillomavirus (HPV) and clinical outcome. Int J Mol Sci 19: E978, 2018.

35. Näsman A, Bersani C, Lindquist D, Du J, Ramqvist T and Dalianis T: Human papillomavirus and potentially relevant biomarkers in tonsillar and base of tongue squamous cell carcinoma. Anticancer Res 37: 5319-5328, 2017.

36. Sajed DP, Faquin WC, Carey C, Severson EA, H Afrogheh A, A Johnson C, Blacklow SC, Chau NG, Lin DT, Krane JF, et al: Diffuse staining for activated NOTCH1 correlates with NOTCH1 mutation status and is associated with worse outcome in adenoid cystic carcinoma. Am J Surg Pathol 41: 1473-1482, 2017. 